\section{W-shaped and U-shaped functions obtained for monoptic and dichoptic disk-disk masking*}

\author{
NAOMI WEISSTEIN \\ Loyola University, Chicago, Illinois 60626
}

For nonmetacontrast (disk-disk) masking, if the outer diameter of the mask equals that of an annulus which produces typical metacontrast functions, $U$ - and $W$-shaped functions will be obtained if (1) the stimuli are viewed dichoptically and monoptically, respectively, and (2) the mask is no more than about 10 times the energy (luminance $X$ duration) of the target. As this energy ratio is approached, both types of functions become monotonic. These shapes suggest that interactions at different visual-pathway loci may be mapped by visual masking functions.

Waveforms obtained in masking experiments have been of two types. In the first type, the strength of masking is monotonic decreasing with respect to increasing intervals between target and mask. (When the target is presented first, these intervals are designated $+\Delta t$; when the mask is presented first, these intervals are designated $-\Delta t$.) Although, if one considers the entire range of $\Delta$ ts over which masking is obtained, the first type of waveform is also $\mathrm{U}$ shaped; the term "U shaped" has been reserved for those waveforms where the masking maximum occurs at some $\Delta t \neq 0$. U-shaped functions have been found, with only a few exceptions, ${ }^{1}$ in metacontrast masking alone, that is, only when a target and mask arc strictly adjacent to each other and in no way superimpose on the same retinal area. It should be noted that U-shaped functions are found in metacontrast only when the target is not too dim or too brief with respect to the brightness and duration of the mask; a rough estimate is that the mask should be no greater than 10 times the energy of the target (Kahneman, 1968: Weisstein, 1968). Energy ratios will be designated $T_{\mathfrak{e}} / \mathrm{M}_{\mathfrak{c}}$, where $T_{\mathfrak{c}}=$ energy of the target and $M_{e}=$ energy of the mask, in foot-lamberts $X$ duration.

The conditions under which the metacontrast effect is found suggest that metacontrast reflects the activity of central mechanisms involved in contour formation. The effect rapidly diminishes when the distance between target and mask borders increases (Alpern, 1953) and when the similarity between target and mask contours decreases (Werner, 1935); and the effect can be obtained dichoptically (Kolers, 1962; Weisstein \& Growney, 1969).

The activity indicated by monotonic functions in backward masking cannot be

\footnotetext{
*Supported by Public Health Service Research Grant No. 8 RO1, EY 00143-02 from the National Eye Institute.
}

as readily specified, since a number of different paradigms lead to such functions, including, as noted above, metacontrast where $T_{e} / M_{c}<.1$. However, there are some masking paradigms yielding monotonic backward masking functions which appear both to be mediated by peripheral mechanisms and to indicate operations on the stimulus that are not concerned with pattern information. These would presumably be brightness or change of brightness operations, in accord with current neurophysiological and psychophysical findings on the locus of light adaptation. In any case, whether or not information processed peripherally can be called brightness information, the masking function is obtained only monoptically if there is an absence of interacting contours (Kahneman, 1968). In general, disk-disk paradigms are used: if the diameters of the masking disks are considerably greater than those of the target disk (i.e.. if $T_{d} / M_{d}$ is small, with $T_{d}$ = diameter of target and $\mathrm{M}_{\mathrm{d}}=$ diameter of mask), then there is no dichoptic masking. Sharp monotonic backward masking functions are obtained in these paradigms, with masking effects diminishing to 0 at ubout $50 \mathrm{msec} \Delta \mathrm{t}$. There may be an enhancement of the target at values of $\Delta t$ greater than $50 \mathrm{msec}$ (Donchin \& Lindsley, $1965)$.

If this kind of analysis of different masking functions is generally correct, then certain predictions follow. First, since metacontrast functions are assumed to reflect activity in contour mechanisms, there is no reason why metacontrast alone should yield U-shaped functions. As long as $\mathrm{T}_{\mathrm{e}} / \mathrm{M}_{\mathrm{c}}>.1$, a number of stimulus conditions should yield a maximum masking effect at some $\Delta t \neq 0$. In particular, in disk-disk masking, where $M_{d}$ $=$ the outer diameter of an annulus known to yield U-shaped metacontrast functions. a similar $U$ should be obtained. Since disk-disk masking will also yield peripheral effects with maximum masking at $\Delta \mathrm{t}=0$, the $U$ may be obscured if the target and mask are presented to the same eye; so this prediction would be made definitely only for dichoptic masking. On the other hand, monoptic metacontrast functions generally have maxima at sizable $\Delta \mathrm{ts}$; so it may be possible, given sufficiently high $T_{e} / M_{e}$, to obtain W-shaped functions in the monoptic case. (The $W$ describes the function across both positive and negative $\Delta$ ts.) This function would have an initial maximum due to peripheral effects and a later maximum due to central effects. The separation between the two troughs would be predicted either from the absence of masking at certain intervals or from an added enhancement effect at certain intervals.

These effects have not been previously reported. It is possible that this is due to the combinations of stimulating conditions typically run. Recall that, in order to obtain $U$ shapes, large $T_{e} / M_{e}$ and $T_{d} / M_{d}$ are needed. In general, masking paradigms which superimpose target and mask have used small $T_{t} / M_{c}$ and $T_{d} / M_{d}$ (Kahneman, 1968). Thus, the following experiment was run using large size and energy ratios.

\section{PROCEDURE}

A disk was used for both target and mask; the masking disk matched the outer diameter of an annulus previously used in metacontrast masking. $\mathrm{T}_{\mathrm{e}} / \mathrm{M}_{\mathrm{e}}$ was varied. This was done to provide a test of the explanation that $\mathrm{U}$ and $\mathrm{W}$ functions have not previously been found for disk-disk masking because low $T_{e} / M_{e}$ was used. If this argument holds, then in this experiment not only should $U$ and $W$ shapes be ubtained with large $T_{e} / M_{e}$ but also, as $T_{\mathrm{c}} / \mathrm{M}_{\mathrm{c}} \rightarrow 0$, both monoptic and dichoptic masking functions should tend toward monotonic functions of $\Delta t$. The specific procedure was as follows.

The study presented a lighted target disk, $1 \mathrm{deg}$ of visual angle, and a lighted I-deg 23-nin masking disk, whose centers were 1 deg from a lighted 4-fL 4-min central fixation cross. In a previous experiment using the same Ss, U-shaped metacontrast functions were obtained with the same target and an annular mask of the same outside diameter (1 deg $23 \mathrm{~min}$ ) when $T_{\mathrm{e}} / \mathrm{M}_{\mathrm{c}}>.0625 .^{2}$ The monoptic maximum occurred at about $80 \mathrm{msec} \Delta t$; the dichoptic maximum occurred at about $40 \mathrm{msec} \Delta t$. The dichoptic function is skewed; there is a considerable amount of masking present at $\Delta t=0$.

Stimuli were transilluminated kodalith slides, presented in a six-channel binocular tachistoscope (Scientific Prototype, Type G) which enables presentation of three fields independently to each eye. Stimuli were presented both monoptically 
and dichoptically in separate sessions in random sequence. For dichoptic viewing, to find the interocular distance and the interocular angle for proper fusion to occur, the target and mask were tumed on for indefinite exposure. The $S$ then adjusted the viewing angle of the right three fields of the tachistoscope and the distance from the right to the left three fields of the tachistoscope until the display appeared stabilized. In addition, two 90-deg prisms for each set of three fields could be adjusted so that the fields for the two eyes were superimposed. When the display was fused, it was turned off, a fixation point was turned on, and this was also fused. Each eye viewed the display through a 2-mm artificial pupil. The masking disk was presented at $16 \mathrm{fL}$ for $16 \mathrm{msec}$ at $27 \Delta$ ts (from -100 to +100 in $10-\mathrm{msec}$ increments, from +100 to +200 in $25-\mathrm{msec}$ increments, and at 250 and $300 \mathrm{msec}$ ), together with a target of one of five energies: $\mathrm{A}=204.8, \mathrm{~B}=128, \mathrm{C}=51.6$, $D=32, E=16$ (foot-lamberts $X$ milliseconds). The energies were varied by decreasing either target luminance and holding target duration at $16 \mathrm{msec}$, or by decreasing target duration and holding target luminance at $16 \mathrm{fL}$, or by decreasing both target duration and target luminance by the same amount. Luminances were decreased by placing Kodak Wratten No. 90 neutral density fulters in front of the slides. Since the variation in masking functions with these ways of varying energy is not of major concern in this report, and since each way of decreasing energy yields the major effects reported here, all data for a particular target energy are combined. The variations in target energy resulted in the following $\mathrm{T}_{\mathrm{e}} / \mathrm{M}_{\mathrm{e}}$ ratios: $\mathrm{A}=.875, \mathrm{~B}=.5, \mathrm{C}=.2$, $\mathrm{D}=.125, \mathrm{E}=.0625$. A ratio of .1 was not specifically tested; Conditions $\mathrm{D}$ and $\mathrm{E}$ were to "bracket" that $\mathrm{T}_{e} / \mathrm{M}_{\mathrm{e}}$ above which U-shaped functions would be found and below which monotonic functions would be found.

For each condition, a complete set of 27 $\Delta$ ts was presented in random order; there were 32 such random orders. There were 15 replications of each energy condition, and the energy conditions were presented in random sequence for each of three Ss. Thus, each $S$ gave 15 responses to each energy condition for each $\Delta t$. All luminances were measured by an SEI Zoomar photometer, and luminance and duration of all stimulus flashes were subsequently monitored by an oscilloscope that displayed the output of photocells placed in each channel of the tachistoscope. Ss were shown, at the beginning of each session, the unmasked target (the standard) for each condition
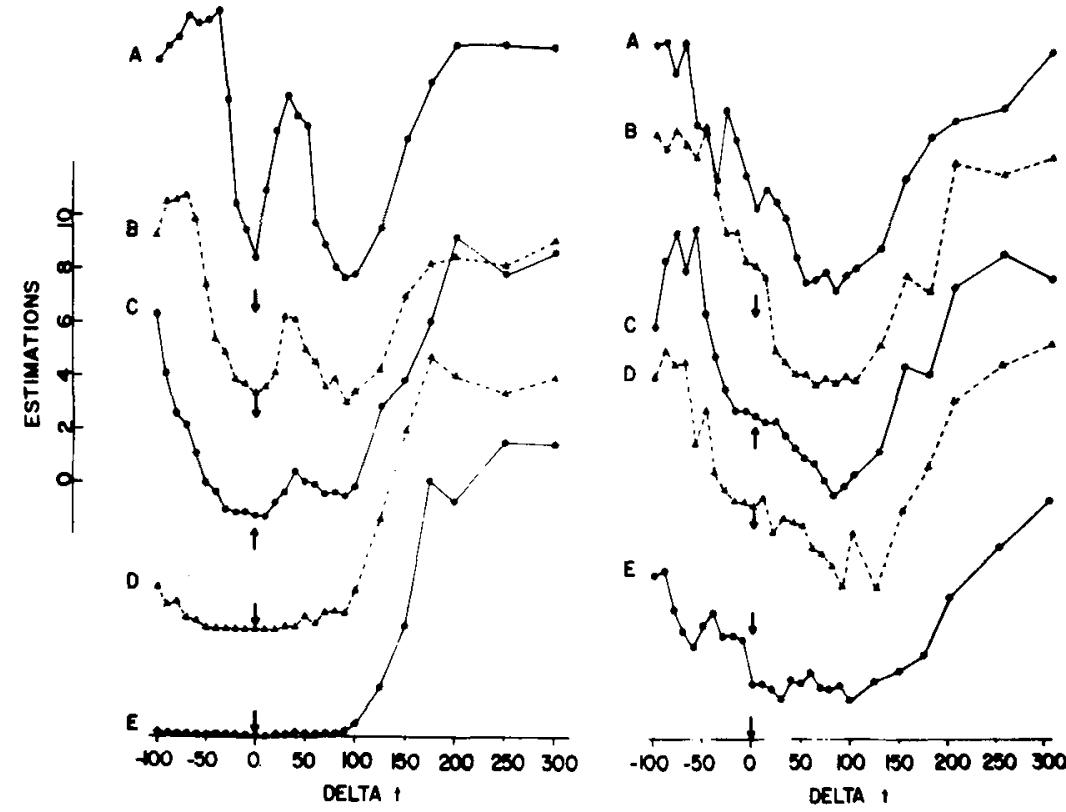

Fig. 1. Magnitude estimations of the completeness (proportion of disk remaining) of a 1-deg lighted target disk when masked by a 1-deg 23-min lighted disk. Target energy decreases from Condition A to Condition E. Each point is a geometric mean of three Ss. For clarity, each function has been offset 2 estimation points from the preceding function. Arrows indicate the point for each function where $\Delta t$ and estimation $=0$. Left side: monoptic viewing; right side: dichoptic viewing.

and were asked to assign to it a number which represented its "completeness" (absence of fragmentation, clearness of contour, etc.). ${ }^{3}$ For each masking presentation Ss judged the completeness of the target in terms of the standard. All Ss assigned the value 10 to the standard. The unmasked target was presented prior to the start of the experiment; it was presented again at any time during the experiment if the $S$ so requested. 4

\section{RESULTS}

The data were transformed into logarithms ${ }^{5}$ and a four-way analysis of variance was then performed: Energy by Eye by Ss by $\Delta t$. The error terms in this analysis are the next-order interaction involving Ss (Edwards, 1968). All main effects (with the exception of Ss, for which there exists no suitable error term) were significant at $\mathrm{p}<.001$. In addition, significant interactions were obtained for Energy by $\Delta t$ (i.e., the shapes are different for the different target energies), for Eyes by $\Delta t$ (i.e., the shapes are different for monoptic vs dichoptic viewing), and for Energy by Eye by $\Delta t$ (i.e., the shapes change over energy conditions differently for monoptic and dichoptic viewing), all at $\mathrm{p}<.01$.

The differences are apparent from Fig. 1. Each point is a geometric mean over three Ss for monoptic and dichoptic and energy conditions separately. (Individual
S's graphs are not shown for brevity; each $\mathrm{S}$ displayed $\mathrm{U}, \mathrm{W}$, and monotonic shapes, so that these are not artifacts of summation across Ss.) Each function has been offset 2 estimation points from the preceding function for clarity.

Three features of these data are especially interesting. First, pronounced W-shaped functions are obtained under monoptic viewing for $T_{e} / M_{e}>.125$. The first troughs in the $W$ do not appear to be the nonmetacontrast analog of what has been called a paracontrast effect, since paracontrast is generally much weaker than the corresponding metacontrast effect, occurs both monoptically and dichoptically, and has a masking maximum at $\Delta t<0$ (Alpern, 1953; Kolers \& Rosner, 1960). Thus, these shapes are not limited to metacontrast paradigms as long as contours are close, $T_{e} / M_{e}$ is not too small (in these data, $.0625<\mathrm{T}_{\mathrm{e}} / \mathrm{M}_{\mathrm{e}}$ ), and dichoptic viewing conditions are used. Finally, as $T_{e} / M_{e} \rightarrow 0$, both dichoptic and monoptic viewing conditions produce monotonic functions of $\pm \Delta t$. This suggests that there may be a considerable number of variations in phase and shapes of masking functions that may have been obscured so far by using small $\mathrm{T}_{\mathrm{e}} / \mathrm{M}_{\mathrm{e}}$, as most previous masking studies have done.

\section{DISCUSSION}

Results are as predicted: If U-shaped metacontrast functions are obtained 

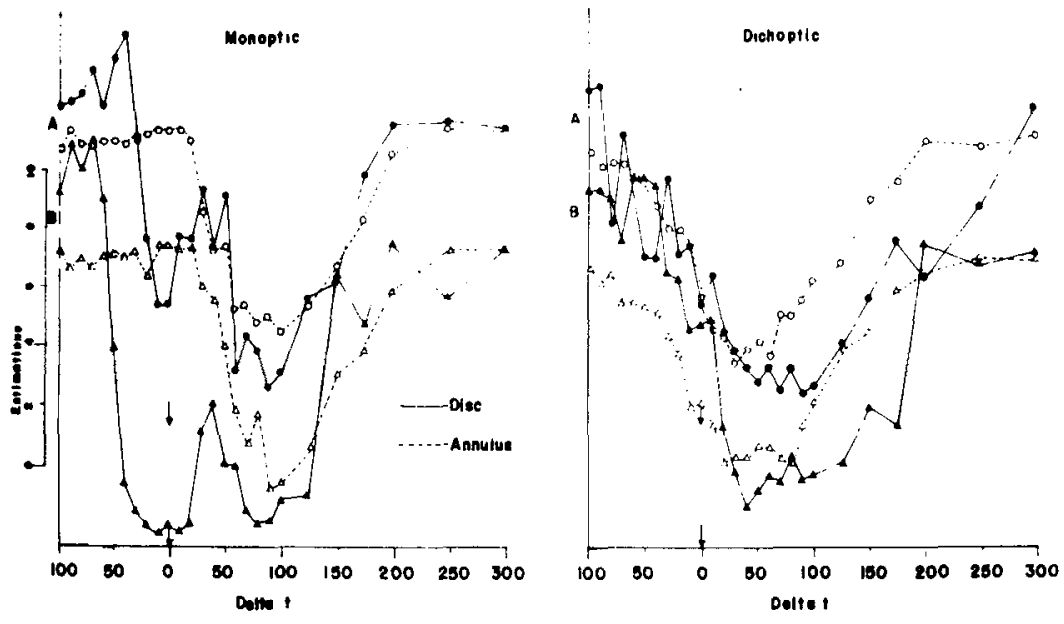

Fig. 2. Magnitude estimations of the completeness (proportion of disk remaining) of a lighted 1-deg target disk when masked by a 1-deg 23-min lighted disk (solid lines) and when masked by an annulus with a 1-deg 1-min inside diam and a 1-deg 23-min outside diam (dotted lines). Target luminance decreases from $12.8 \mathrm{fL}$ in Condition A (circles) to $8 \mathrm{fL}$ in Condition B (triangles). Each point is a geometric mean of three Ss. For clarity, the $B$ functions have been offset $i$ estimation points from the $A$ functions. Arrows indicate the point for each function where $\Delta t$ and estimation $=0$.

dichoptic nonmetacontrast paradigms with close target and mask contours should also yield U-shaped functions, and they do. Similarly, if masking by flashes yields sharp monotonic backward masking functions, then W-shaped functions should be obtained with the same stimuli under monoptic conditions, and they are. The first trough of the $W$ attains its minimum at $\Delta \mathrm{t}=0$, and in other respects resembles peripheral masking by flashes (Spering. 1965), including an enhancement effect at large negative $\Delta \mathrm{ts}$. However, the second trough of the $W$ does not match the dichoptic $U$; the descent of the dichoptic $($; clearly starts well before the ascending arms of the first trough of the monoptic $W$. This may be explained by the differences in phase which are obtained for monoptic and dichoptic metacontrast. A comparison between annular masking from a previous experiment, ${ }^{2}$ in which the same Ss, energy ratios, target. response indicator, and experimental procedure were used, and the monoptic disk-masking data from this experiment show that, while there are some differences in the functions, the $\Delta t$ at which maximum masking begins is the same for Energy Condition A and about $20 \mathrm{msec}$ earlier for Condition B. This is shown in Fig. 2, which presents data for Energy Conditions $A$ and $B$ over the same three $S s$ in the same manner. This time, however, A and B refer only to conditions of decreasing $T_{\mathrm{e}} / \mathrm{M}_{\mathrm{e}}$ through decreases in target luminance, and only five estimations per $S$ were taken. The $A$ and $B$ ratios are the same, i.e., $\mathrm{A}=.875, \mathrm{~B}=.5$. The dotted-line functions are anmular masking. i.e., metacontrast masking; the solid-line functions are disk masking. The dichoptic functions show a greater disparity in phase for Conditions $A$ and $B$ than do the $U$ parts of the monoptic functions. But here again. Condition $\mathrm{A}$ is more nearly similar in phase than is Condition B. The phase differences for dichoptic functions are opposite in direction from the monoptic functions. The reason for these differences in plase and the indication that phase differences increase as $T_{e} / M_{c}$ becomes smalter are matters for further investigation. In any case. it is clear that the second trough in the W corresponds quite well with monoptic metacontrast data.

What conclusions can be drawn from these data? There exists no theory of visual masking which can account for the variety of effects obtained through the action of a single mechanism. For instance. any general "confusion hypothesis" (sce Weisstein, 1908, for a discussion of this lype of hypothesis) which assumes that masking is some function of the confusion attendant on the presentation of two stimuli close in time is entirely inadequate to deal with the various shapes which call be obtained under different masking conditions. Or all "erasure" type explanations which assume that at $\Delta t=0$ the larget and mask are perceived simultaneously and, therefore, are both perccived but that at some critical $+\Delta t>0$ the target has not yet been "read in" 10 some central storage and the mask can thus "replace" it, cannot with ease handle a second maximum masking effect, since the number of operations that produce two maxima are greater than the number of operations assumed by the model.

On the other hand, explanations that see different masking functions as a result of different specific operations in the visual system, the locus and characteristics of which depend on the features of a stimulus being coded, are sufficiently flexible to explain a number of shapes. Such an explanation is part of a more general theoretical framework which sees perception as a process consisting of a series of stepwise transformations on visual signals, with different afferent orders responsible for transforming and coding different features of a stimulus. On theoretical grounds, feature-specific theories of visual processing are suggested by artificial intelligence programs for pattern recognition which make use of property analysis, and they are suggested empirically by the neurophysiological findings that single units at various locations in the visual system fire to particular features of stimuli and to no others.

Not only can feature-specific explanations for visual masking identify different operations in the visual system by different visual masking functions, but also such explanations are conveniently related to fairly specific types of neural circuitry. The value of establishing the relation between psychophysical functions and neural circuitry has been discussed by Weisstein (1969). The locus of the masking eftect, the type of loss of information involved, and the temporal characteristics of the neural response will all depend on the features of the target and mask and the conditions of their presentation.

In summary, no general theory of visual masking may exist precisely because different masking functions are mediated by different mechanisms. At the same time. all masking functions may provide information of a similar order, namely, the identification of mechanisms in terms of feature-specific neural responses. This would be the unifying basis of theories of visual masking: neural explanations which assume that different features of a stimulus are processed at different times and locations in the visual system.

In terms of this kind of theoretical framework, then, masking functions could serve as temporal "maps" of different operations in the visual system. In particular, for the data reported here, the different operations seem likely to be contour interactions in the case of $\mathrm{U}$-shaped functions and first brightness contour interactions in the case of U-shaped functions, and first brightness, then contour interactions in the case of W-shaped functions. 


\section{REFERENCES}

ALPERN, M. Metacontrast. Journal of the Optical Society of America, 1953, 43, 648-657.

BLANC-GARIN, J. Divers aspects de l'interference dans le phénomène de masquage latéral. Vision Research, 1968, 8, 509-523.

DONCHIN, E., \& LINDSLEY, D. B. Retroactive brightness enhancement with brief paired flashes of light. Vision Research, 1965, 5, 59-69.

EDWARDS, A. L. Experimental design in psychological research. New York: Holt, Rinehart, \& Winston, 1968.

FITZGERALD, R. E. Visual masking as a function of the observer's task. Paper read at meetings of the Midwestern Psychological Association, 1970.

KAHNEMAN, D. Method, findings, and theory in studies of visual masking. Psychological Bulletin, 1968, 70, 404-423.

KOLERS, P. A. Intensity and contour effects in visual masking. Vision Research, 1962, 2, 277-294.

KOLERS, P. A., \& ROSNER, B. S. On visual masking (metacontrast): Dichoptic observation. American Journal of Psychology, $1960,73,2-21$.

MAYZNER, M. S., TRESSELT, M. E. \& HELFER, M. S. A provisional model of visual information processing with sequential inputs. Psychological Monograph Supplements, 1967, 2(7, Whole No. 23).

SCHILLER, P. H., \& GREENFIELD, A. Visual masking and the recovery phenomenon.
Perception \& Psychophysics, 1969, 6, 182-184.

SPERLING, G. Temporal and spatial visual masking: I. Masking by impulse flashes. Journal of the Optical Society of America, $1965,55,541-559$.

STEVENS, S. S. Duration, luminance, and the brightness exponent. Perception \& Psychophysics, 1966, 1, 96-100.

WEISSTEIN, N. A Rashevsky-Landahl neural net: Simulation of metacontrast. Psychological Review, 1968, 75, 494-521.

WEISSTEIN, N. What the frog's eye tells the human brain: Single cell analyzers in the human visual system. Psychological Bulletin $1969,72,157-176$.

WEISSTEIN, N., \& GROWNEY, R. L. Apparent movement and metacontrast: $A$ note on Kahneman's formulation. Perception \& Psychophysics, $1969,5,321-328$.

WERNER, H. Studies on contour: I. Qualitative analyses. American Journal of Psychology, $1935,47,40-64$.

\section{NOTES}

1. $U$ shapes have been reported for trains of adjacent letters (Mayzner, Tresselt, \& Helfer, 1967); it is not clear whether this is a metacontrast effect. Kolers (1962) did get U-shaped functions with nonmetacontrast black stimuli; however, he repeatedly failed to find this effect with flashes of light. Finally, Schiller and Greenfield (1969) obtained a U shape with disk-disk masking flashes for a mask with a diameter .04 deg larger than the target, but not with masks of larger diameters.

2. N. Weisstein, in preparation.

3. In previous metacontrast experiments with these same Ss, both brightness and completeness judgments were required. While other investigators (Fitzgerald, 1970; Blanc-Garin, 1968) have reported that they obtain differen masking functions, depending on the type of judgment required, in these metacontrast experiments no significant differences were found between the two types of judgments. Brightness judgments did tend to be somewhat more variable, however, so in this experiment only completeness judgments were used.

4. The judgment of masking of each target energy in terms of the value 10 allows direct comparison of functions with varying target energies. In addition, Ss were asked to make magnitude estimations of the unmasked target with reference to an unmasked target (superstandard) at $16 \mathrm{fL}, 16 \mathrm{msec}$; these latter estimations could be used to scale the magnitude estimations between conditions, so as to obtain an absolute measure of masking. The scaling was done by multiplying estimations for a particular condition by the fraction (number assigned to the unmasked target when compared to superstandard/10). In the results reported here, the shapes of the masking functions are of direct interest; thus, results are reported in terms of unscaled values.

5. It is assumed that these magnitude estimations are distributed log normally. See Stevens (1966).

(Accepted for publication July 2, 1970.) 